\title{
Re-Launch des Service-Portals für Patienten
}

In Österreich sind mehr als zwei Millionen Menschen von chronisch entzündlichen Darm- und Hauterkrankungen sowie Rheuma betroffen. Das von AbbVie ins Leben gerufene Portal www.therapietreue-info.at bietet ihnen Zugang zu Informationen rund um den Umgang mit Rheuma, Morbus Bechterew, Morbus Crohn, Colitis ulcerosa, Plaque Psoriasis oder Acne inversa. Für die Betroffenen ist hohe Eigenverantwortung und Selbstmanagement wichtig für einen positiven Therapieverlauf. Mit dem nunmehrigen Re-Launch des Service-Portals unterstützt AbbVie die Gesundheitskompetenz der Patienten.

Quelle: Presseaussendung AbbVie
Das neu strukturierte OnlinePortal www.therapietreue-info.at wurde noch übersichtlicher gestaltet und mit zusätzlichen Inhalten aufgewertet. Patienten finden auf der Website jetzt unter anderem auch Tipps zur Vorbereitung für das Gespräch und Kontrolluntersuchungen beim Arzt. Eine bessere Kenntnis der Erkrankung ermöglicht dem Betroffenen gemeinsam mit dem Arzt wichtige Entscheidungen im Rahmen der Therapie zu treffen.

Traude Schaffer, Präsidentin der Österreichischen Rheumaliga: „Die Plattform bietet uns Betroffenen wertvolle Unterstützung. Man erhält fundierte und zugleich ver-

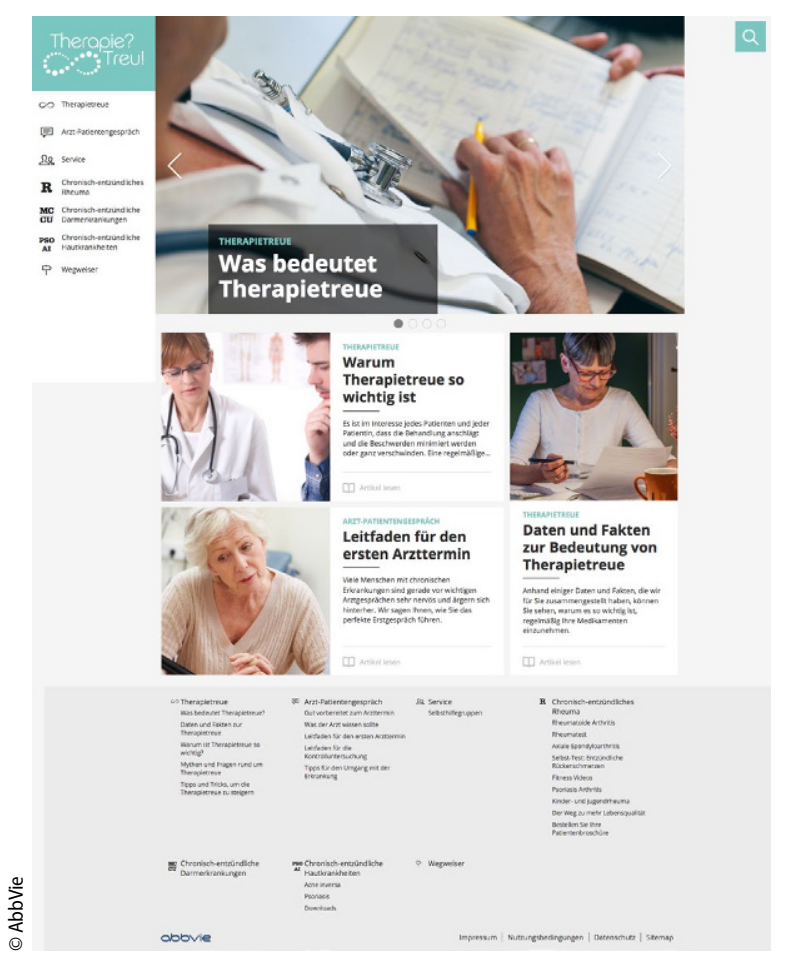

ständlich aufbereitete Informationen über Erkrankungen, Symptome, Diagnosestellung und Behandlungsmöglichkeiten und darüber hinaus Hilfestellungen für den Alltag. Man kann sich so wesentlich besser auf Gespräche mit dem Arzt vorbereiten und ihm gegenübertreten."

Ergänzt wird das praxistaugliche Serviceangebot durch Kontaktlisten zu Selbsthilfegruppen, einen Wegweiser zu Fachärzten und Apotheken aber auch einen Plan von öffentlichen Toilettenanlagen in Wien etc.

\section{Behandlungserfolg durch Compliance}

Ein weiterer neuer Bereich der Website beschäftigt sich mit dem Thema Therapieerfolg. Hier geht es um die verbindliche Vereinbarung von Verhaltensregeln mit dem Arzt, nicht nur im Hinblick auf die Einnahme der Medikamente sondern auch betreffend Ernährungsumstellung, Bewegung und Änderungen des Lebensstils. Wie man "Therapietreue" einfach in den eigenen Alltag integrieren kann, wird anhand von Beispielen aufgezeigt.

Diese Bewusstseinsbildung beim Betroffenen ist von großer Bedeutung. Priv.-Doz. Dr. Wolfgang Weger (Universitätsklinik für Dermatologie und Venerologie, Graz) erklärt dazu: „Das Therapieziel muss realistisch und erreichbar sein und sollte gemeinsam mit dem Patienten festgelegt werden. Denn das aktive Miteinbeziehen des Betroffenen in diese Entscheidung wirkt sich positiv auf seine Therapietreue aus. Das Ziel kann sich im Laufe einer Behand- lung ändern und muss deshalb regelmäßig angepasst werden. Hat ein Betroffener gegenüber einer Behandlung ernste Bedenken, ist das Nicht-Einhalten vorprogrammiert; Ängste müssen ernst genommen, besprochen und mögliche Alternativen diskutiert werden."

Seitens AbbVie sieht man das Portal als einen weiteren Schritt zur Aufklärung in Sachen chronischer Erkrankungen und zur Verbesserung der Behandlung: „Die Steigerung der Gesundheitskompetenz in der Öffentlichkeit und bei Betroffenen sowie die Förderung des optimalen Selbstmanagements im Rahmen ihrer Therapie sind wichtige Anliegen für uns", betont Mag. Ingo Raimon, General Manager von AbbVie in Österreich: „Wir entwickeln laufend unabhängig von der Bereitstellung unserer innovativen Arzneimittel Initiativen, um die Lebensqualität der Patienten weiter zu verbessern. Die Plattform www.therapietreueinfo.at ist ein wichtiger Schritt in diese Richtung."

\section{Weitere Informationen:}

www.therapietreue-info.at www.abbvie.at

rheuma plus $2017 \cdot 16: 128$ https://doi.org/10.1007/s12688017-0148-9

(c) Springer-Verlag GmbH Austria 2017 